\title{
Changing Concepts of Cerebrospinal Fluid Hydrodynamics: Role of Phase-Contrast Magnetic Resonance Imaging and Implications for Cerebral Microvascular Disease
}

\author{
Stavros Michael Stivaros and Alan Jackson \\ Imaging Science and Biomedical Engineering, The Medical School, University of Manchester, Manchester, United Kingdom
}

\begin{abstract}
Summary: Phase-contrast magnetic resonance imaging (PCMRI) or flow-sensitive MRI can be used to noninvasively measure intracranial vascular and CSF flow. Monro-Kellie homeostasis is the complex compensatory mechanism for the increase in intracranial blood volume during systole. Through PC-MRI techniques, our understanding of Monro-Kellie homeostasis and the associated intracranial hydrodynamics has greatly improved. Failure of this homeostatic mechanism has been implicated in a wide range of cerebral disorders, including vascular and Alzheimer's dementia, late-onset depression, benign and secondary intracranial hypertension, communicating and normal pressure hydrocephalus, and age-related white matter changes. The most common mode of homeostatic failure is
\end{abstract}

due to vascular disease with decreased cerebral arterial compliance. This has wide-reaching implications in the investigation of patients with cerebral vascular disease. Here we discuss the role of PC-MRI in the study of cerebral hydrodynamics and the current understanding of Monro-Kellie homeostasis in both healthy and disease states. Quantitative assessment of the changes in this homeostatic mechanism using PC-MRI has important implications in the development of biomarkers of vascular involvement in disease with application in diagnosis, treatment planning, phenotype identification, and outcome assessment in clinical trials. Key Words: Phase-contrast MRI, cerebrospinal fluid, hydrodynamics, homeostasis, hydrocephalus.

\section{INTRODUCTION}

Our understanding of the mechanisms involved in the production, flow, and absorption of cerebrospinal fluid (CSF) has changed significantly over the last 10 to 20 years. Of particular interest is the understanding of changes in CSF flow that occur in response to the inflow of blood into the cerebral circulation during cardiac systole. Because the contents of the skull are incompressible, the transient increase in blood volume during systole induces a complex compensatory mechanism now known as Monro-Kellie homeostasis. This homeostatic mechanism not only compensates for increased intracranial systolic blood volume, but results in significant smoothing of the systolic/diastolic pressure differences to which the brain is exposed.

Failure of Monro-Kellie homeostasis has been implicated in the etiology and pathogenesis of a wide range of cerebral disorders, including vascular dementia, Alzheimer's disease $(\mathrm{AD})$, late-onset depression, benign intracranial hypertension, secondary intracranial hypertension, communicating hydrocephalus, normal pressure hydrocephalus, and age-related white-matter changes. The mechanism may fail for a number of reasons, but the most common mode of failure results from vascular disease in which there is a decrease in cerebral arterial compliance. This being a cardinal feature of cerebral atheroma, the Monro-Kellie homeostatic mechanism has wide-reaching implications in the investigation of patients with cerebral vascular disease.

Here we will review the MRI techniques used to study cerebral hydrodynamics and then review the current understanding of Monro-Kellie homeostasis in health and disease.

\section{PHASE CONTRAST MAGNETIC RESONANCE IMAGING}

Much of our understanding of Monro-Kellie homeostasis has been derived from the application of

\footnotetext{
Address correspondence and reprint requests to: Professor Alan Jackson, BSc, MB ChB, FRCR, Imaging Science and Biomedical Engineering, The Medical School, University of Manchester, Oxford Road, M13 9PT UK. E-mail: Alan.Jackson@ Manchester.ac.uk.
} 
phase-contrast magnetic resonance imaging (PC-MRI) techniques. ${ }^{1-5}$ To measure regional flow velocity, PCMRI uses the change in the phase of the MR signal that occurs when fluid flows in a static magnetic gradient. When blood or CSF flows through a magnetic gradient, the phase of the magnetic signal will change in a manner proportional to its velocity. ${ }^{6}$

Most PC-MRI systems use a bipolar gradient in a process called flow encoding. Two equal but opposite gradient pulses are applied to the tissue. Each of these will shift the phase of spins within the tissue. In stationary tissues, the effects of the second pulse will exactly reverse the effects of the first. However, fluid moving through the tissue in the period between the pulses will experience a net phase shift proportional to its velocity. This technique can be applied to high-resolution images, and data can be collected using respiratory or cardiac gating. The net effect is that the technique provides a noninvasive measurement of flow velocity which has a high spatial and temporal resolution and is both accurate and reproducible. ${ }^{7}$

The data are reproduced in the form of four-dimensional ( $x, y, z$, and time) images in which the intensity of the image is a direct measure of flow velocity. The same data can also be used to produce intensity images that are highly sensitive to structures containing flow and so can be used to identify their boundaries. The bulk flow of fluid through a blood vessel or CSF space can be assessed by defining a region of interest conforming to the vessel outline on intensity images and then integrating the volume within this region of interest on the flow velocity map. If the sequence is cardiac-gated and the heart rate is known, then the blood flow through an individual vessel can be calculated in milliliters per minute. $^{7,8}$

PC-MRI is sensitive to a range of velocities, which can be specified by choosing a maximal expected velocity known as the velocity-encoding value $\left(V_{\mathrm{enc}}\right)$. Different velocity-encoding values can be used to optimize the accuracy of measurements in structures with widely differing flow rates. ${ }^{7,9}$ In vivo and in vitro studies have shown PC-MRI to be reliable ${ }^{10-12}$ and highly reproducible. $^{7}$

Application of PC-MRI in the brain allows routine measurement of blood flow rates within all the major arteries, veins, and CSF spaces. Combination with cardiac gating allows the operator to extract time-resolved volume-flow curves for individual vessels (FIG. 1).

PC-MRI data can be acquired using two-dimensional or three-dimensional acquisition sequences. In practice, time constraints lead most investigators to use a combination of two-dimensional measurements from specific anatomical points of interest. A typical example is the study presented by Bateman ${ }^{14}$ regarding the relationship between cerebral blood flow and idiopathic intracranial hypertension. This used a retrospectively cardiac-gated phase-contrast sequence of TR $29 \mathrm{~ms}$, TE $7 \mathrm{~ms}$, flip angle $30^{\circ}$, slice thickness $6 \mathrm{~mm}$, matrix size 192/512, and field of view $20 \mathrm{~cm}$. The $V_{\text {enc }}$ values were $20 \mathrm{~cm} / \mathrm{s}$ for the venous structures and $75 \mathrm{~cm} / \mathrm{s}$ for the arterial ones. The
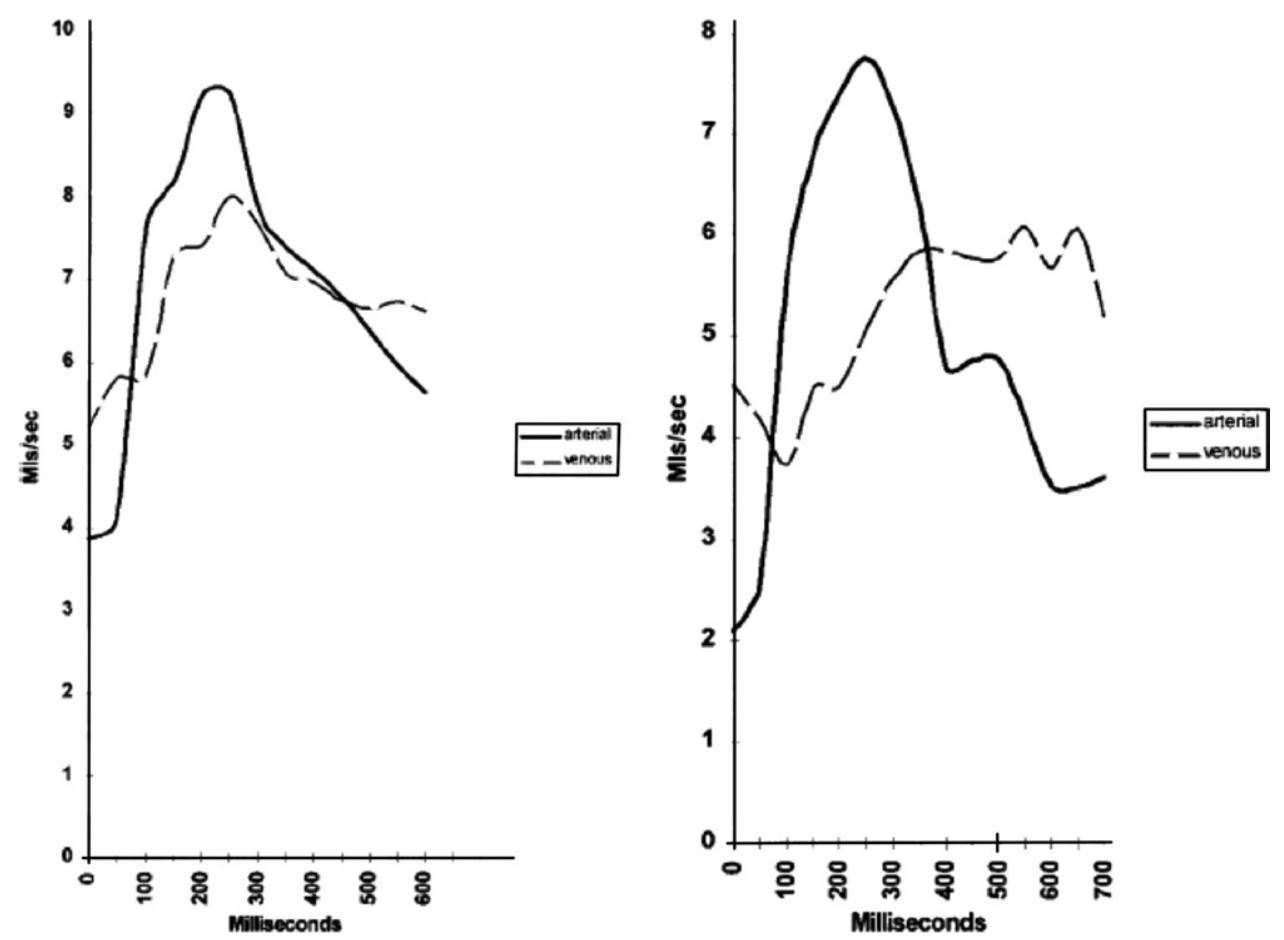

FIG. 1. Graphs showing arterial inflow and venous flow in the superior sagittal sinus territory in a healthy patient (left) and in a patient with cerebral atrophy (right) obtained using PC-MRI modified from Bateman. ${ }^{13}$ 

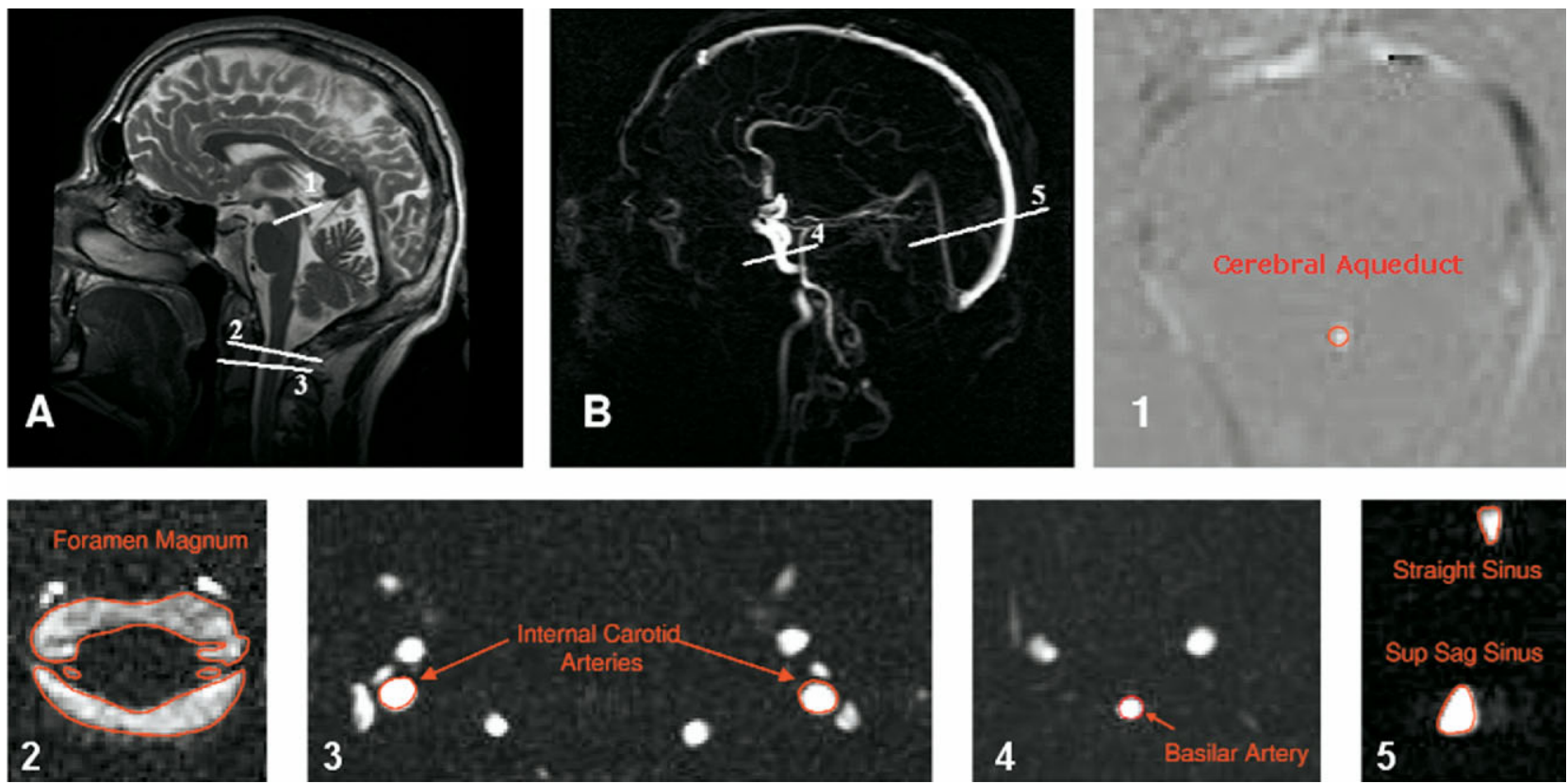

FIG. 2. Sample PC-MRI images taken during the development of a simple electrical equivalence model of Monro-Kellie homeostasis. ${ }^{19}$ (A) Sagittal T1 scout image to determine flow acquisition level and oblique axis for cerebral aqueduct and foramen magnum CSF flow and internal carotid artery blood flow. (B) Phase-contrast angiogram used to determine acquisition level for the basilar artery, straight and superior sagittal sinuses. (1-5) Typical velocity-encoded MR images of the vessels and CSF flow: 1, cerebral aqueduct; 2, foramen magnum; 3 , internal carotid arteries; 4, basilar artery; 5 , straight sinus and superior sagittal sinus.

plane of the sections was designed to intersect the superior sagittal sinus (SSS) $2 \mathrm{~cm}$ above the confluence of the sinuses and pass through the basilar artery and the intracavernous internal carotid arteries. ${ }^{14,15}$

This protocol was validated in vitro and in vivo with error rates of the order of $5 \%$ and both intra- and interobserver variability described as low to negligible. ${ }^{16-18}$ Figure 2 shows an example of the typical protocol used at our institution, allowing measurement of blood flow through the carotid and basilar arteries, SSS, straight sinus, and jugular vein, as well as CSF flow through the cerebral aqueduct and foramen magnum.

\section{CSF PRODUCTION AND ABSORPTION}

\section{The bulk flow theory}

Cerebrospinal fluid (liquor cerebrospinalis) is the clear fluid occupying the space between the arachnoid and pial layers of the meninges. It is a saline solution containing $\sim 0.3 \%$ plasma proteins with a concentration of $15-40$ $\mathrm{mg} / \mathrm{dL}$, depending on the sample site. ${ }^{20}$ CSF contains microglial cells, acts as a cortical buffer, and supports transport of metabolites and neurotransmitters. CSF also occupies the ventricular system of the brain and bathes the spinal cord, as well as the brain, which in effect floats within the CSF with a resultant effective weight reduction of $\sim 97 \%$. $^{21}$ Displacement of CSF in response to linear and shearing forces acts as a buffer to reduce the effects of mechanical forces on the brain.
CSF is produced at a rate of $\sim 500 \mathrm{~mL}$ per day, so that the entire CSF volume $(\sim 150 \mathrm{~mL})$ is replaced three times during any 24-hour period. It is commonly taught that CSF is produced exclusively by the choroid plexus, which is found in the lateral ventricles and the roof of the third and fourth ventricles. CSF secretion causes a continuous net flow from the lateral ventricles through the interventricular foramina, into the third ventricle, and then via the cerebral aqueduct into the fourth ventricle. From here it flows through the lateral foramina of Luschka and the median foramen of Magendie, through the cerebromedullary cistern, down the spinal cord and over the cerebral hemispheres. From here, traditional teaching has it that it is absorbed into the venous system via the arachnoid (pacchonian) granulations, which lie mainly over the vertex of the prosencephalon.

This conventional view of CSF production and absorption at the arachnoid granulations is known as the bulk flow theory, wherein the driving force of the flow of CSF is the pressure differential caused by the production of CSF at the choroid plexus being greater than the pressure at the absorption site at the arachnoid granulations. Hence, the intracranial pressure is dependent upon the relationship between the production and absorption of CSF. The bulk flow theory effectively explains the dilatation of the ventricles in patients with obstructive hydrocephalus caused by, for example, tumors at the level of the third ventricle. However, problems exist with this theory because, as we will see, the main site of CSF 
absorption is not the arachnoid granulations but the capillaries of the central nervous system. ${ }^{21}$

Modern concepts of CSF production and absorption. Even as early as 1914, Dandy ${ }^{22}$ highlighted significant problems with the bulk flow theory, stating that if there were a blockage at the pacchonian granulations, such an obstruction would not cause a higher pressure in the dilated ventricles than in the subarachnoid space but would instead dilate the subarachnoid space. He hypothesized that CSF absorption is in fact a diffuse process occurring everywhere within the capillaries of the subarachnoid space. Other observations also cast doubt on the bulk flow theory, most notably the absence of pacchonian granulations in children until the closure of the fontanels and the effectiveness of third ventriculostomy for the treatment of communicating hydrocephalus. If absorption of CSF is occurring purely in the arachnoid granulations, then producing a communication between the third ventricle and the subarachnoid space should have no effect on abnormal ventricular dilatation.

It is now generally accepted that CSF is produced far more widely than was previously believed. Interstitial fluid is produced by the cerebral capillaries, and there is exchange of this fluid across the pial membrane into the subarachnoid space, where the interstitial fluid "adequately substitutes [for] the CSF." 21 Indeed, so similar are the CSF and interstitial fluids that the two cannot be differentiated in any way save by their location. Not only are the brain capillaries important for a degree of CSF production (a relatively small degree, admittedly, and not forgetting the more significant contribution of the choroid plexus), but also for the majority of CSF absorption. Again, in a manner similar to other organs bathed in fluid, the brain capillaries actively take up plasma proteins and other molecules from the CSF; this causes an absorption of interstitial fluid and CSF into the capillary and hence into the venous outflow. ${ }^{21,23}$

\section{CSF FLOW AND PULSATION}

The demonstration that CSF is widely absorbed throughout the subarachnoid space does not help explain the dilatation of the ventricles that occurs in patients with communicating hydrocephalus. If resorption of CSF occurs widely throughout the subarachnoid space, then ventricular dilatation can be explained only by gross overproduction of CSF from the choroid plexus at such a rate that the resorption mechanism is unable to cope. This is clearly not the case, because these patients respond to third ventriculostomy.

Initial insight into the mechanism that underlies ventricular dilatation came from early PC-MRI studies in patients with normal pressure hydrocephalus (NPH). NPH is a clinical syndrome ${ }^{24-26}$ of mental deterioration, gait disturbance and urinary incontinence seen in elderly individuals. Ventricular dilatation is often associated with ischemic white-matter lesions. Early clinical studies failed to demonstrate any increase in ventricular pressure; however, it later became apparent that, although the mean pressure within the ventricular system is normal, there are marked and abnormal variations across the cardiac cycle. This led investigators to apply PC-MRI techniques to study the relationship between pulsatile flows of blood and CSF within the cranial cavity.

\section{MONRO-KELLIE HOMEOSTASIS}

The Monro-Kellie hypothesis states that "if the skull is intact, then the sum of the volumes of the brain, CSF and intracranial blood is constant."27-29 Hence, if one of these elements within the cranium increases in volume it must occur at the expense of the volume of the other two. PC-MRI has enabled elucidation of the events that occur during the normal cardiac cycle. ${ }^{30}$

In normal healthy individuals, systolic expansion of the basal cerebral arteries produces a pressure wave within the subarachnoid CSF, which causes an outflow of CSF through the foramen magnum into the compliant spinal CSF space, equivalent to $\sim 50 \%$ of the increase in intracerebral blood volume. ${ }^{1,21}$ Similarly, with diastolic relaxation of the artery the CSF flows back into the cranium again through the foramen magnum. In this way, there is flow of CSF back and forth through the foramen magnum.

The systolic CSF pressure wave is also transmitted to the major dural venous sinuses by systolic expansion of the arachnoid granulations. ${ }^{30,31}$ The effect of this is that the systolic pressure wave is dissipated by the formation of CSF and venous pulsatility and largely bypasses the cerebral circulation. In addition, the elastic artery walls absorb part of the energy of the systolic arterial pulse wave, which is then released during diastole to maintain constant capillary flow. This is known as the windkessel effect. Constancy of cerebral perfusion pressure is also maintained by transient systolic increases in venous backpressure within the brain, due to direct compression of cortical surface veins by the systolic pulse wave in the subarachnoid CSF space. This combination of processes maintains a constant perfusion pressure and flow in the cerebral capillary bed, despite the major pressure changes seen between systole and diastole.

In summary, therefore, distension of the intracerebral arteries due to the windkessel mechanism, along with the CSF pressure wave enabling venous dilatation, causes decreased vascular resistance and enables continuous high blood flow within the brain capillaries during the complete cardiac cycle. This mechanism is illustrated in Figure 3 as modified from Greitz. ${ }^{21}$

Closer examination of Figure 3 shows that during presystole no pressure gradients are present in the brain. 


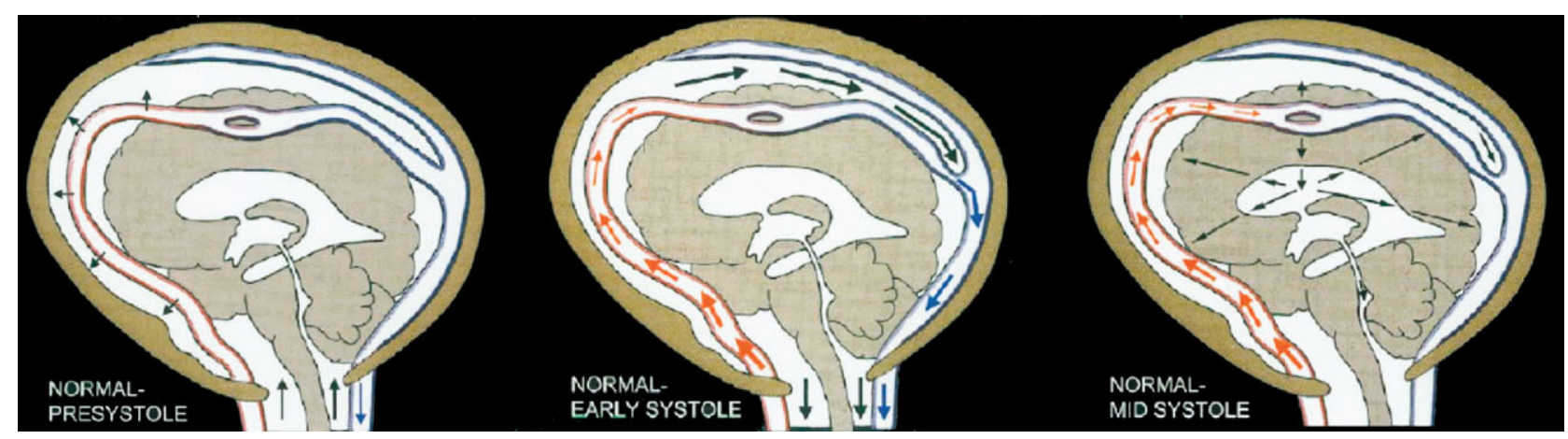

FIG. 3. Normal intracranial hydrodynamics. The relative thickness of the arrows in the artery (red) indicates the magnitude of pressure. The relative thickness of the arrows in the venous system (blue) and subarachnoid space indicates the magnitude of flow. Modified from Greitz. $^{21}$

CSF flows into the cranial cavity from the spinal canal. In early systole, the systolic pulse wave causes large arterial expansion, with a concomitant and significant dampening of the arterial pulse pressure that is transmitted to the entire subarachnoid space. This expansion causes volume conduction of CSF, compressing the outlets of the cortical veins and increasing systolic blood flow in the venous sinus. Simultaneously, the arterial expansion causes a large systolic expulsion of CSF into the compliant spinal canal. By midsystole, the small, dampened pulse wave in the artery (FIG. 3, thin arrows) is transmitted into the brain capillaries. This causes a slight brain expansion and a transmantle stress of normal magnitude. ${ }^{21}$

The intracranial extracerebral arterial expansion is of the order of $1.5 \mathrm{~mL}$ and is composed of the stroke volumes at the foramen magnum and within the venous sinuses. ${ }^{21}$ The volume increase due to brain capillary expansion, however, is much smaller (on the order of $0.03 \mathrm{~mL}$ ) and is directed toward the ventricular system. It is thus equal to the volume of CSF pulsed through the cerebral aqueduct. ${ }^{21,32-34}$

\section{ABNORMAL CSF FLOW AND COMMUNICATING HYDROCEPHALUS}

As already discussed, simple obstruction of CSF absorption at the level of the arachnoid granulations (in contrast to obstruction of CSF flow by, for example, tumor at the level of the cerebral aqueduct) will not cause the ventricular dilatation seen in communicating hydrocephalus. It is now widely accepted that ventricular dilatation in communicating hydrocephalus results from increased transmission of the systolic pulse wave into the cerebral capillary bed. Indeed, it has been suggested that communicating hydrocephalus should be renamed "increased capillary pulsation hydrocephalus." 21

The initial cause of this increased transmission of the systolic pulse wave into the brain might be multifactorial. The most common etiology appears to be a decrease in the compliance of the intracerebral arteries. This might reflect vessel wall disease due to atheroma, or a reduced ability of the vessels to dilate due to decreased compliance in the surrounding CSF space. Whatever the initial mechanism, systolic/diastolic pressure variations are less effectively dampened and this pressure difference is transmitted to the cerebral capillary bed.

Increased capillary pulsatility will cause brain dilatation during systole with an associated increase in intraventricular pressure. This leads to a net transmantle pressure difference between the ventricular system and the subarachnoid space with subsequent ventricular dilatation. This process sets in motion a series of secondary effects. These include continuous compression of the veins along their course within the subarachnoid space causing decreased vascular compliance and reduced blood flow. This in turn increases cerebral venous pressure, leading to diminished perfusion pressure and resultant reduced CSF absorption. The mechanisms involved in the generation of communicating hydrocephalus are illustrated in Figures 4 and 5, which have associated explanatory figure legends from Greitz. ${ }^{21}$

\section{NORMAL PRESSURE HYDROCEPHALUS}

Normal pressure hydrocephalus is a form of communicating hydrocephalus that is clinically characterized by the triad of dementia, gait disturbance, and urinary incontinence in elderly individuals. It can be considered as a subtype of communicating hydrocephalus, believed to have a coherent etiology and pathogenesis.

PC-MRI has shown significant reduction in cerebral vascular compliance in patients with NPH. ${ }^{13}$ Bateman $^{13}$ showed a decrease in the arteriovenous delay of 53\% compared with normal subjects, combined with a $29 \%$ drop in blood flow through the SSS compared with the normal. That study demonstrated a clear dichotomy of vascular changes between the superficial and deep venous drainage systems. The deep venous drainage system, represented by drainage through the straight sinus, 


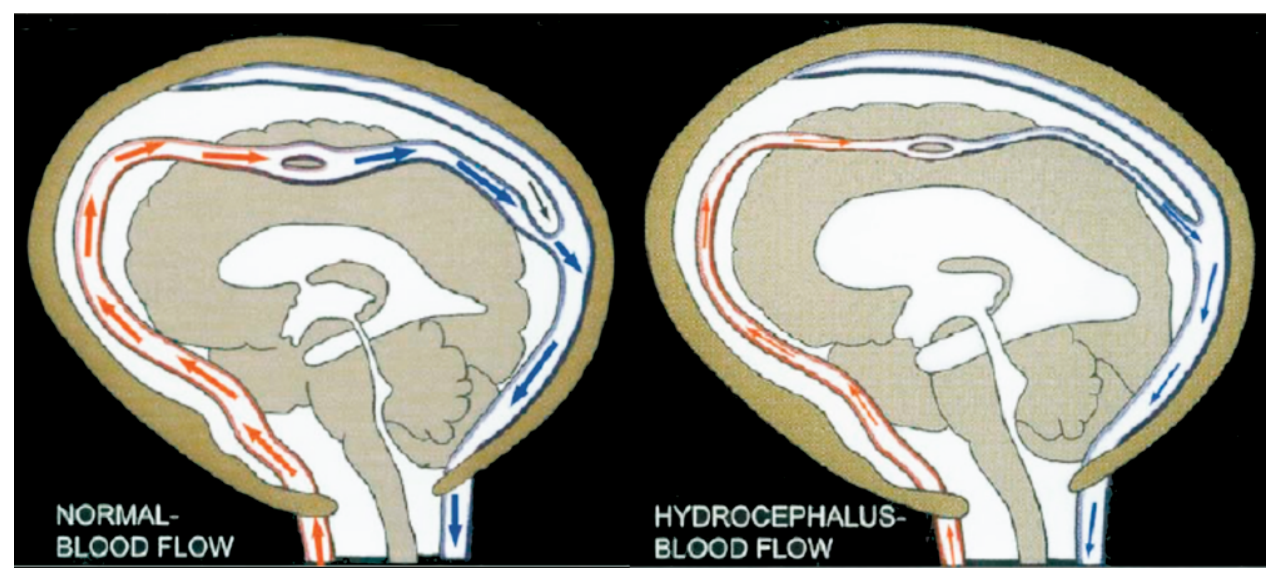

FIG. 4. Cerebral blood flow in healthy individuals and in communicating hydrocephalus. Maintenance of normal intracerebral blood flow is by a combination of the arterial windkessel mechanism and the venous outflow resistance that keeps the cerebral veins distended maintaining high normal blood flow. The venous outflow resistance is caused by a small positive intracranial pressure and is increased during systole. The venous outflow resistance is a mandatory prerequisite what is called the waterfall phenomenon (i.e., the pressure drop occurring from the cortical veins to the venous sinus). In communicating hydrocephalus, the increased transmantle pulsatile stress and the ventricular dilatation compress the cerebral veins and capillaries in their entire length. This significantly increases the vascular resistance and decreases the blood flow. The venous outflow resistance is reduced and the normal pressure drop from the subarachnoid space to the venous sinus disappears. This phenomenon cannot be explained by the bulk flow concept, because a pressure drop caused by a CSF obstruction at the arachnoid granulations would increase the pressure drop from the subarachnoid space to the sinus, not decrease it. The reduced venous outflow resistance facilitates collapse of the compressed capacitance vessels, which further decreases cerebral blood flow. Modified from Greitz, ${ }^{21,35}$ with permission.

showed increased pulsatility despite the presence of significantly reduced pulsatility in the SSS. ${ }^{13}$

This finding suggests a functional dichotomy between the deep and superficial venous drainage systems, possibly reflecting the considerable differences between them in terms of exposure to pressure from surrounding CSF. The reduction in vascular compliance results in turn in a reduction of the volume of CSF exiting the foramen magnum during systole. Increased capillary pulsatility is believed to increase inward expansion of the brain against the ventricles with a consequent increase in the rate of CSF flow through the cerebral aqueduct. Mea- surement of aqueduct flow has been proposed as a biomarker to predict eventual response to shunting in these patients.

\section{MECHANISM OF VENTRICULAR EXPANSION IN COMMUNICATING HYDROCEPHALUS}

There are several theories as to the cause of ventricular expansion in communicating hydrocephalus and NPH. Early researchers suggested that the ventricles dilated due to increased resistance to flow in the cerebral aqueduct; however, Bateman ${ }^{13}$ has shown that this is not the

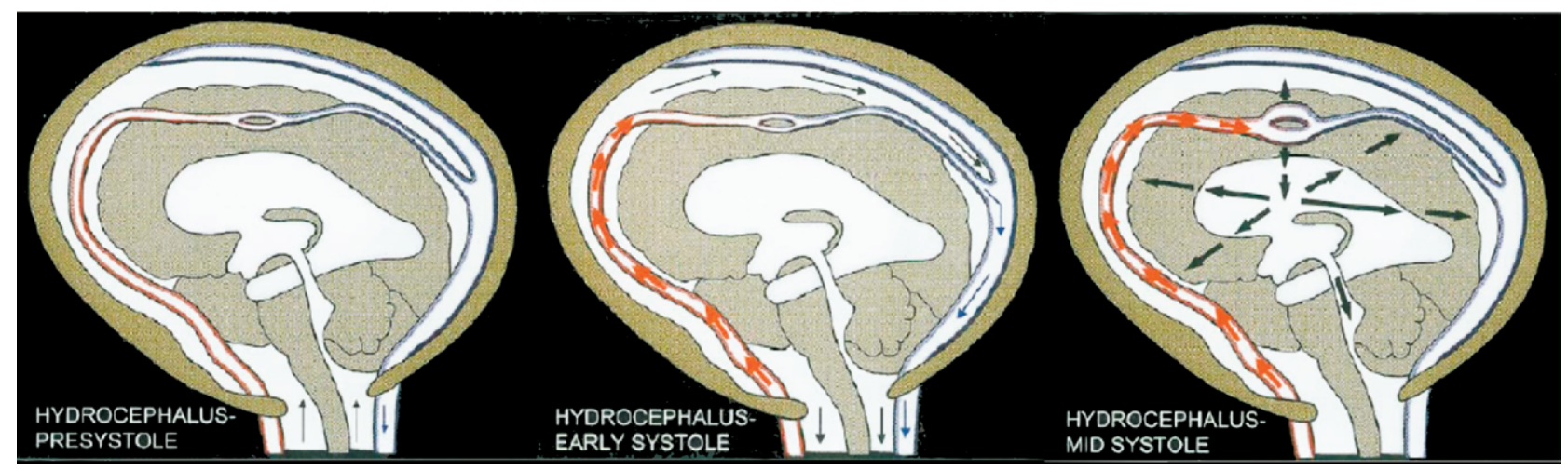

FIG. 5. Hydrodynamics of communicating hydrocephalus. In presystole, the intracranial compliance is decreased. By early systole, the decreased intracranial compliance restricts the arterial expansion, so little or no damping of the pulse pressure occurs in the artery. Due to decreased volume conduction of CSF, the systolic flows in the dural sinus and in the subarachnoid space at the foramen magnum are decreased. Venous outflow resistance is decreased and capacitance vessels collapse. The total vascular resistance is increased. By midsystole, the high pulse pressure in the artery (thick arrows) is transmitted undampened into the brain capillaries, giving rise to a large capillary expansion, an increased CSF pulse pressure, a large systolic flow in the aqueduct, an increased transmantle stress, and ventricular dilatation. The increased transmantle stress compresses the intracranial veins in their entire length, which in turn decreases intracranial compliance, increases vascular resistance and decreases cerebral blood flow. Modified from Greitz, ${ }^{21,35}$ with permission. 
case and that the aqueduct provides little resistance to flow. Greitz ${ }^{4,21,32-34,36}$ suggests that as the brain expands during capillary pulsation the expansion is directed toward the ventricles. This results in an increase in the intraventricular pulse pressure and the hyperdynamic CSF flow which is observed within the cerebral aqueduct.

The question must be asked, why there is expansion of the ventricular system and compression of the brain? This can be explained due to the self-compression of the brain against the incompressible fluid within the ventricular system during each systole. Due to Pascal's law (i.e., the fluid pressure at all points in a connected body of an incompressible fluid at rest, which are at the same absolute height, are the same, even if additional pressure is applied on the fluid at some place), the pressure within the ventricles must equal that within the expanding brain. The brain has a high plasticity and can compensate for volume shifts by chronic reductions in interstitial fluid and cerebral blood volume leading to gradual reductions in size. In contrast, however, Bateman ${ }^{13}$ and Hakim and colleagues ${ }^{37-39}$ have suggested that due to the changes in compliance seen in the SSS and straight sinus, there are larger arterial pulsations in the deep parenchyma of the brain leading to shear stresses, as seen in dementia and atrophy, with resultant parenchymal atrophy and compensatory expansion of the ventricle.

\section{IDIOPATHIC INTRACRANIAL HYPERTENSION AND SECONDARY INTRACRANIAL HYPERTENSION}

The cause of idiopathic intracranial hypertension (IH) (also referred to as benign intracranial hypertension or pseudotumor cerebri) is unknown. It is characterized by raised intracranial pressure with normal CSF composition in the absence of hydrocephalus or intracranial mass lesion. Secondary intracranial hypertension (IH) describes patients with an identical clinical syndrome wherein the increase in intracranial pressure can be demonstrated to result from venous outflow obstruction, most commonly related to dural sinus thrombosis. In such cases, time-of-flight, or post-contrast magnetic resonance venography, or computed tomographic (CT) venography are useful to visualize filling defects or stenoses in the venous sinuses $^{40-43}$ (FIG. 6).

It has been suggested that idiopathic IH results from a failure of cerebral vascular autoregulation causing increases in cerebral blood flow and blood volume with resultant IH. Three groups of investigators have demonstrated elevated blood flow $\sim 50 \%$ above normal in idiopathic $\mathrm{IH}$, but others, using contrast enhanced techniques, have demonstrated lesser elevations or no change. ${ }^{14,43,44}$ Bateman $^{14}$ demonstrated an average arterial inflow of 55\% above that in a normal control group

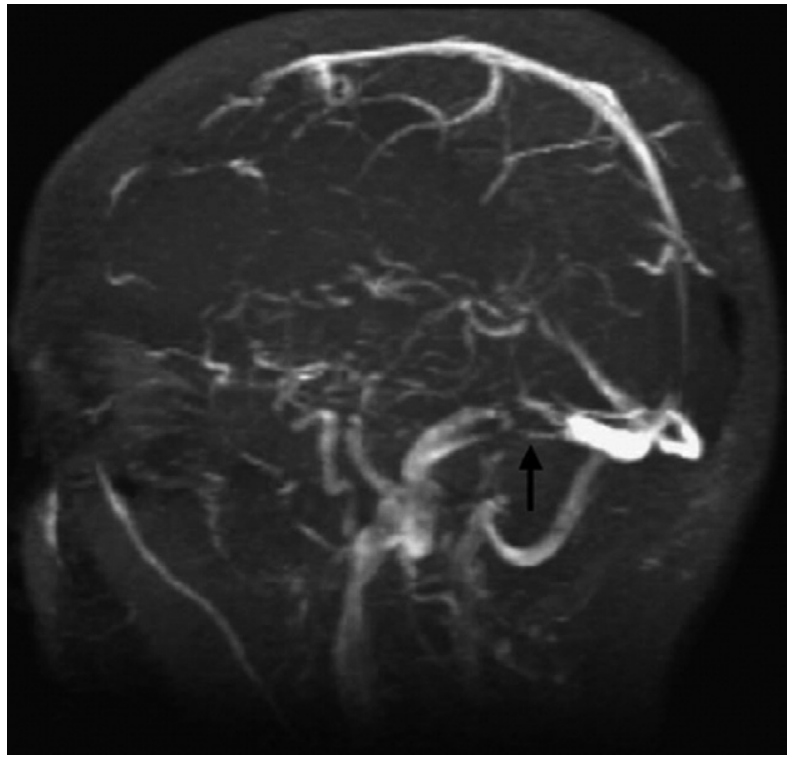

FIG. 6. Magnetic resonance venogram showing a loss of flow in the posterior part of the sagittal sinus and thrombosed proxima transverse sinus (arrow) in a 51-year old woman with intracranial hypertension. Reprinted from Bateman, ${ }^{14}$ with permission.

(1320 $\mathrm{mL} / \mathrm{min} P=0.0001)$; however, the venous outflow was seen to be lower than expected (SSS flow $32 \% \pm 7$ and straight sinus flow $9 \% \pm 2$, compared with $47 \% \pm 6$ and $14 \% \pm 35$ in the control group) suggesting the development of collateral venous outflow. Indeed Bateman ${ }^{14}$ has suggested that "hyperemic intracranial hypertension may be a better descriptor in the subset of patients who have idiopathic IH with raised cerebral blood flow."

In secondary IH, occlusion of the venous sinus (FIG. 6) elevates flow resistance within the sinus in question. This produces an initial increase in venous back pressure and reduced blood flow in the areas of the brain drained by the sinus. The reduction in blood flow will trigger an autoregulatory dilatation of pial arterioles, leading to an increase in arterial flow and consequently a further elevation of venous pressure. ${ }^{14,41}$ Bateman $^{14}$ demonstrated increased collateral drainage in patients with secondary IH due to SSS obstruction with a reduction in SSS flow by $35 \%$ compared with normal subjects $(P=0.0001)$. The percentage of the total inflow drained by the SSS of these patients was $30 \% \pm 8$, compared with $47 \% \pm 6$ in the control group $(P=0.0001) .{ }^{14}$

\section{MICROVASCULAR ANGIOPATHY}

Cerebral microvascular disease is commonly seen with normal aging, and the incidence of white matter lesions and lacunes increases exponentially after the age of $65 .{ }^{45,46}$ Histologically, aging is associated with evidence of cerebral arteriosclerotic microvascular disease, often referred to as microvascular angiopathy 
(MVA). ${ }^{47-49}$ Low-grade MVA is characterized by histological findings of increased tortuosity and irregularity in small arteries and arterioles (Grade 1). ${ }^{50}$ As these changes progress (Grade 2), arteriolar vessel walls show thickening, which eventually leads to the classical onionskin appearance and, in late stages, necrosis within the wall. Local gliosis occurs along with lacunes ranging from small old cystic infarcts, small hematomas and dilated Virchow-Robin spaces. ${ }^{50,51}$

MVA is difficult to assess in vivo and a measure of white matter lesion load (as reflected by T2-weighted hyperintensities in deep white matter, basal ganglia, and periventricular areas on MRI scans) is commonly used as a potential biomarker for MVA in imaging-based studies. ${ }^{52,53}$ Patankar et al., ${ }^{54}$ from work in our laboratory, have recently shown a close relationship between MRIvisualized abnormal dilatation of Virchow-Robin spaces in the basal ganglia, and a diagnosis of vascular dementia in elderly patients.

In a recent study ${ }^{55}$ we hypothesized that the reduction in microvascular compliance caused by MVA would result in detectable changes in CSF flow in the cerebral aqueduct, due to increased passage of the systolic pulse wave into the cerebral capillary bed. We tested this hypothesis in a group of patients with late onset depression, in which there is considerable evidence that treatment resistance and other features of intractable disease result from vascular insult due to MVA. We hypothesized that the reduction in arteriolar compliance would lead to a reduction in the delay between the arterial and CSF systolic pulses and a narrowing of the systolic pulse wave in the cerebral aqueduct, reflecting a reduction in the dampening mechanism within the arterial tree. These hypotheses were supported by the study, ${ }^{55}$ suggesting that disturbances of cerebral hydrodynamics can be used to provide a generic biomarker of microvascular disease in patients with MVA. Recent findings in our laboratory (unpublished data) support this suggestion with the demonstration of abnormal CSF aqueductal flow patterns in elderly normal volunteers with high levels of vascular risk factors, compared with control subjects with low risk.

The demonstration of a breakdown in Monro-Kellie homeostasis has a number of potentially important implications. First, it suggests that measurements of CSF hydrodynamics can be used to generate quantitative biomarkers of microvascular disease. Second, it suggests that the breakdown of Monro-Kellie homeostasis should be considered as part of the pathogenetic mechanism in cerebral vascular diseases characterized by MVA or reduced arterial compliance.

This is of particular importance in the investigation of elderly patients with dementia. Vascular dementia and $\mathrm{AD}$ used to be considered mutually exclusive disorders but there is compelling evidence that the two commonly coexist (what is called mixed dementia). ${ }^{56}$ Vascular dementia is the most common cause of dementia in the fifth to seventh decade. Although it represents a range of clinical syndromes due to different forms of vascular insult, it is thought to result most commonly from extensive MVA. Risk factors traditionally associated with vascular dementia (such as hypertension, dyslipidemia, atherosclerosis, atrial fibrillation, coronary artery disease, diabetes, obesity, and smoking) are now known to also play a significant part in the genesis of $\mathrm{AD} .^{57-59}$ Clinically, it is common to find features of both disorders in an individual patient. The presence of both Alzheimer and vascular pathology is a common finding at post mortem. ${ }^{60}$

The proportion of people with $\mathrm{AD}$ who have vascular risk factors is at least the same as, and often higher than, that in the healthy age-matched population. Rates of hypertension are estimated at in excess of $70 \%,{ }^{61}$ concomitant clinically significant cerebrovascular disease in $30 \%,{ }^{62}$ and untreated hypercholesterolemia in $10 \% .^{63}$ Small-scale studies have shown symptomatic improvement in patients with $\mathrm{AD}$ in response to nimodipine ${ }^{64,65}$ and ventriculoperitoneal shunting. ${ }^{66}$ The use of nimodipine was based on the theory that AD interferes directly with cerebral calcium metabolism; and the use of shunting was based on the hypothesis that it would improve the clearance of waste materials from CSF. However, these observations could also be explained by failure of Monro-Kellie homeostasis in patients with dementia and any such failure could contribute to the onset and severity of cognitive impairment. As yet no studies of CSF pulsatility in patients with dementia have been published, although it has been demonstrated that there is loss of normal vascular compliance in the arterial tree in $\mathrm{AD} .^{67}$

\section{LEUKOARAIOSIS}

Widespread introduction of CT and MRI has led to the recognition that abnormal areas of high signal on T2weighted images are common in the older population and are directly related to increasing age. These lesions within the white matter, often referred to as leukoaraiosis (see FIG. 7), are also associated with vascular risk factors, including hypertension, diabetes mellitus, and cardiac disease. ${ }^{46}$ They are also seen in increasing numbers in patients with vascular dementia and in NPH. ${ }^{46}$ There is considerable evidence that these lesions are ischemic in origin, and it is likely that their etiology is multifactorial.

Irrespective of cause, one finding in these cases is of severe perivenular collagenosis with noninflammatory thickening of the walls of the veins. It has been hypothesized that these changes cause the veins to become less compliant, increasing local venous backpressure with local loss of cerebral perfusion. In addition, the obstruction 


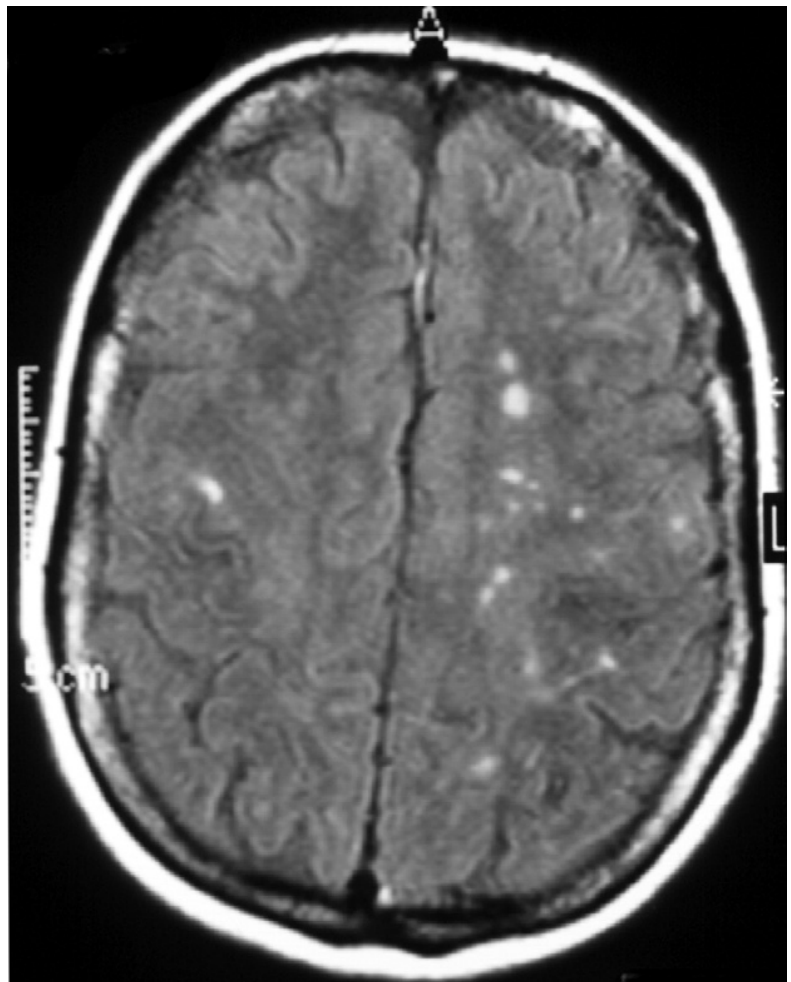

FIG. 7. T2-weighted MRI scan demonstrating moderate leukoaraiosis.

of these venous outflow channels leads to a propagation of the pulse wave from the venous structures to the capillaries and arterioles, further worsening the already diminished cerebral blood flow and capillary hypertension and again causing decreased resorption of CSF. On this basis, it has been suggested that leukoaraiosis is indicative of ischemic cerebral damage due to increased cerebral capillary pulsatility. Indeed, Bateman ${ }^{1}$ has suggested the term pulse-wave encephalopathy to describe the cerebral injury caused by increased capillary pulsation.

\section{RECENT ADVANCES IN CSF MODELING}

Modern theories implicate the breakdown of the autoregulatory system in the pathogenesis of a wide range of diseases, including communicating hydrocephalus, ${ }^{21}$ $\mathrm{NPH},{ }^{13,68}$ secondary $\mathrm{IH},{ }^{15}$ leukoaraiosis, ${ }^{1}$ and neurodegenerative and mixed dementias and other cerebral atrophic disorders. Suggested modes of failure include, for example, changes in arterial compliance and loss of the windkessel effect leading to increased capillary pulsatility in communicating hydrocephalus; decreased arteriolar resistance and increased cerebral blood flow in idiopathic $\mathrm{IH}$; increased venous resistance in secondary $\mathrm{IH}$; and reduced venous compliance in NPH. ${ }^{13}$

There are problems with all of these hypothesized mechanisms, in that the varying sets of phenomenological observations regarding arterial, venous, and CSF flow patterns are commonly open to differing interpretations. As described above in previous work from our laboratory, Naish et al. ${ }^{55}$ examined the transmission of the systolic pulse wave into the cerebral capillaries in elderly patients with treatment-resistant depression. The study demonstrated an increased aqueductal flow volume implying increased cerebral pulsatility with a decrease in the latency between arteriolar and aqueductal systole. This was interpreted as a potential biomarker for de-

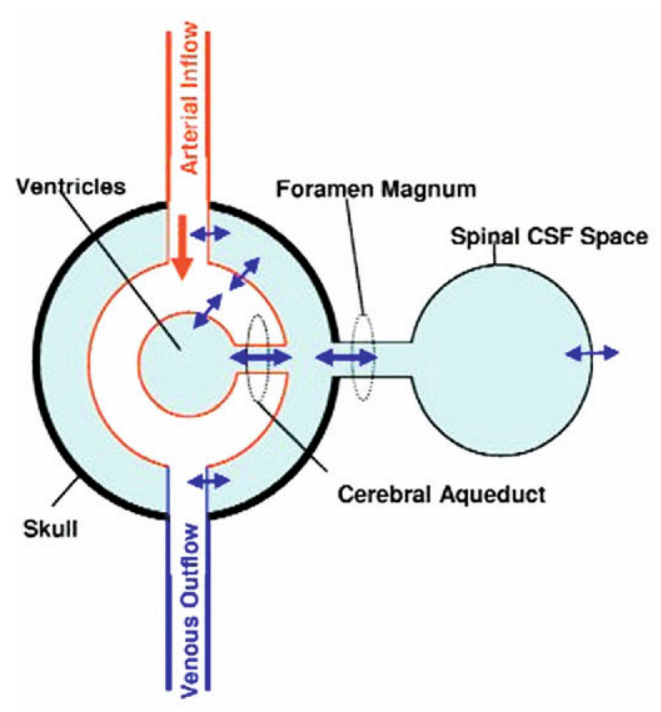

(a)

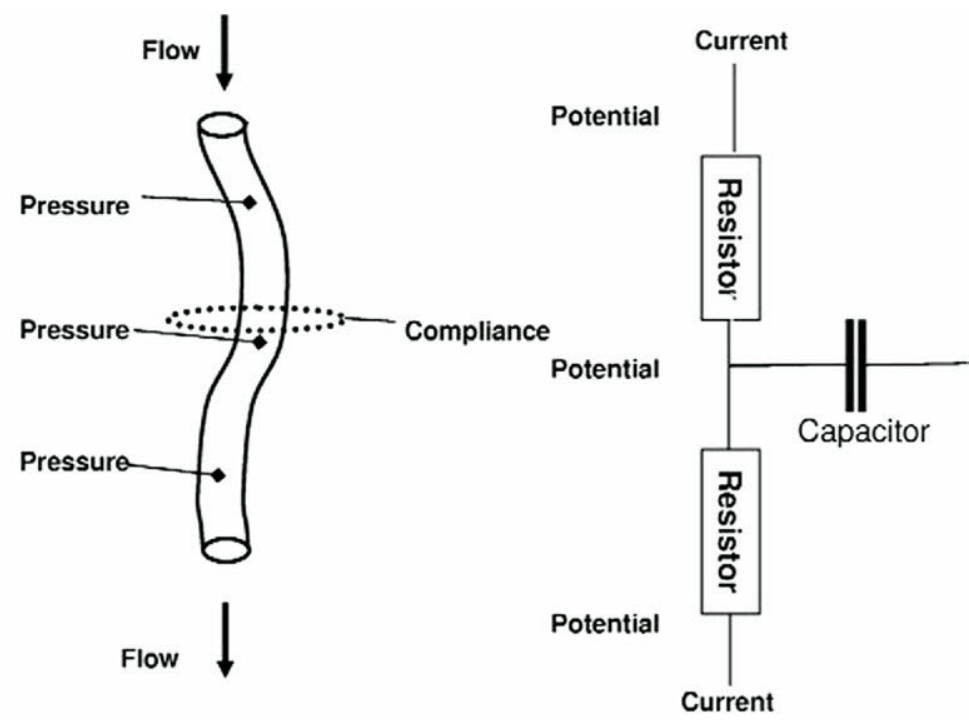

(b)

FIG. 8. Bulk parameter model of Monro-Kellie homeostasis using the analogy of an electronic circuit. (a) Simplified anatomical model on which the circuit is based and (b) the analogy between current and flow. Both systems can be characterized by analogous first-order differential equations. Reproduced from Kim et al., ${ }^{19}$ with permission. 
creased arteriolar compliance due to microvascular angiopathy; however, other interpretations of these findings are possible, such as increasing brain pulsatility due to a change in the compliance of the brain substance (due to ischemic white matter changes) with a normal vascular pulse pressure. Due to a combination of the complexities of this autoregulatory mechanism, the variations in measurement techniques and interpretation by different groups, and limitations in MR flow techniques and imaging times, such phenomenological observations are becoming increasingly unsatisfactory.

In view of these limitations, there is a clear need for a quantitative physiological model of the autoregulatory model underpinning Monro-Kellie homeostasis to facilitate analysis of PC-MRI data. As is elaborated elsewhere, ${ }^{19}$ any such model must achieve the following goals:

1) Enable direct comparison between different study designs.

2) Allow identification of pathogenic abnormalities interfering with autoregulatory mechanisms that would not be susceptible to simple phenomological observation.

3) Allow testing and validation of specific data driven analysis techniques to identify minimum required data sets to support valid conclusions regarding clinical diagnosis and pathogenetic mechanisms in specific disease processes.

Kim et al., ${ }^{19}$ therefore have proposed a bulk parameter model using the analogy of an electrical circuit (FIGS. 8 and 9).

This model describes the interactions between intracranial arterial and CSF flow patterns observed in normal individuals, and is the first of its kind to be tested with genuine data. It allows for direct proportional estimation of seven parameters:
1) Arterial compliance
2) Brain compliance
3) Ventricular compliance
4) Venous compliance
5) Arterial impedance
6) Brain impedance
7) Impedance at the cerebral aqueduct

The model has been validated in normal individuals and is able to accurately predict venous outflow patterns given a limited number of input parameters. This suggests that the model is valid, ${ }^{19}$ although it remains overly simplistic, because it treats the intracranial space as a single black box. Such a model will require further development to account for anatomical variations, such as the suggested differences between superficial and deep

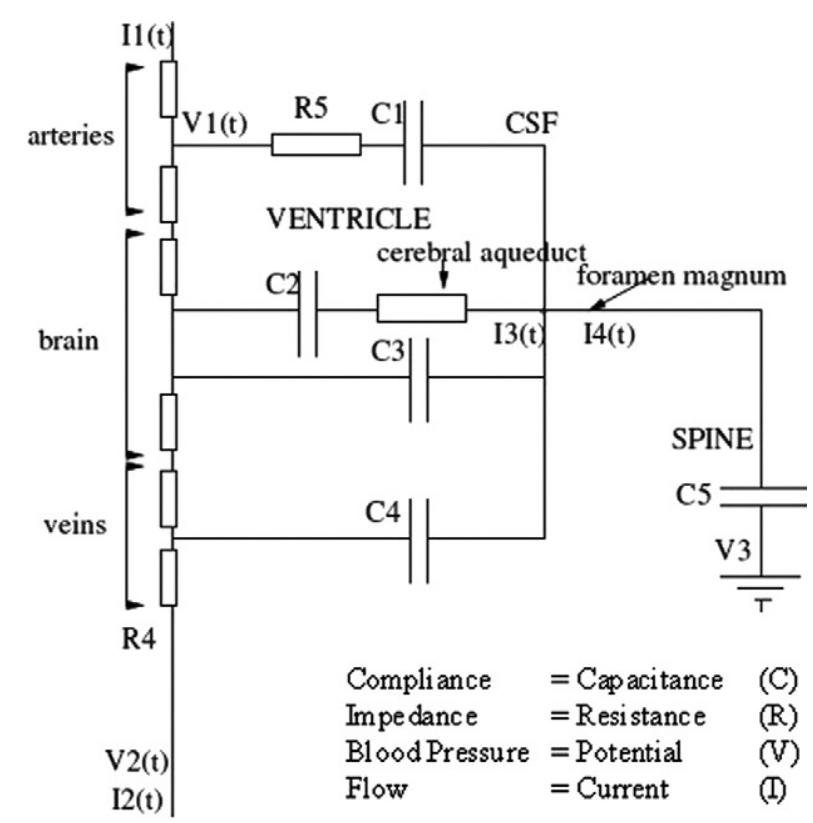

(a)

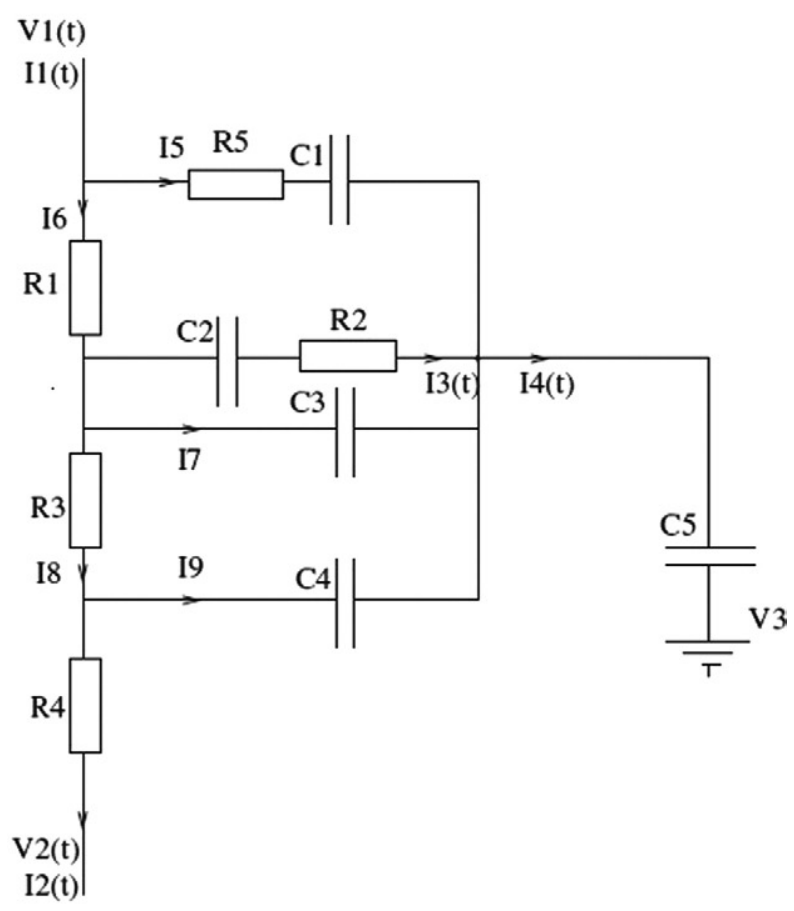

(b)

FIG. 9. Electrical circuit of the biological model of Monro-Kellie homeostasis. (a) Analogous electrical circuit; (b) combination of redundant components and specification of all current path variables. Reproduced from Kim et al., ${ }^{19}$ with permission.

venous drainage and the effect of restriction of CSF flow between the supra and infratentorial spaces. Nevertheless, the development of a model of this type gives a framework within which PC-MRI data can be used to test specific biological hypotheses regarding the measurable parameters listed here. 


\section{SUMMARY}

We have seen that the development and application of PC-MRI over the past 10-20 years has completely changed our understanding of the physiology of CSF production, flow, and resorption. The elucidation of the mechanisms underlying Monro-Kellie homeostasis has identified a complex and delicate autoregulatory mechanism that can be impaired by a wide range of pathological changes. Current research suggests that abnormalities of Monro-Kellie homeostasis might be involved in the pathogenesis not only of disorders characterized by changes in cerebral flow (such as idiopathic IH and secondary $\mathrm{IH}$ ), but also in disorders conventionally believed to be neurodegenerative (such as AD and leukoaraiosis). It seems likely that quantitative assessments of the disturbances in Monro-Kellie homeostasis will become important biomarkers of vascular involvement in a wide range of disease states, with application in diagnosis, treatment planning, phenotype identification, and outcome assessment in clinical trials.

\section{REFERENCES}

1. Bateman GA. Pulse-wave encephalopathy: a comparative study of the hydrodynamics of leukoaraiosis and normal-pressure hydrocephalus. Neuroradiology 2002;44:740-748.

2. Baledent O, Gondry-Jouet C, Stoquart-Elsankari S, et al. Value of phase contrast magnetic resonance imaging for investigation of cerebral hydrodynamics. J Neuroradiol 2006;33:292-303.

3. Baledent O, Gondry-Jouet C, Meyer ME, et al. Relationship between cerebrospinal fluid and blood dynamics in healthy volunteers and patients with communicating hydrocephalus. Invest Radiol 2004;39:45-55.

4. Greitz D, Wirestam R, Franck A, et al. Pulsatile brain movement and associated hydrodynamics studied by magnetic resonance phase imaging: the Monro-Kellie doctrine revisited. Neuroradiology 1992;34:370-380.

5. Levy LM, Di Chiro G. MR phase imaging and cerebrospinal fluid flow in the head and spine. Neuroradiology 1990;32:399-406.

6. Hashemi RH, Bradley WG. MR Angiography. In: MRI: the basics. Mitchell CW, editor. Baltimore: Williams \& Wilkins, 1997:280283.

7. Spilt A, Box FM, Van der Geest RJ, et al. Reproducibility of total cerebral blood flow measurements using phase contrast magnetic resonance imaging. J Magn Reson Imaging 2002;16:1-5.

8. Ho SS, Chan YL, Yeung DK, et al. Blood flow volume quantification of cerebral ischemia: comparison of three noninvasive imaging techniques of carotid and vertebral arteries. AJR Am J Roentgenol 2002;178:551-556.

9. Gill RW. Measurement of blood flow by ultrasound: accuracy and sources of error. Ultrasound Med Biol 1985;11:625-641.

10. Bakker CJ, Kouwenhoven M, Hartkamp MJ, et al. Accuracy and precision of time-averaged flow as measured by nontriggered $2 \mathrm{D}$ phase-contrast MR angiography, a phantom evaluation. Magn Reson Imaging 1995;13:959-965.

11. Bakker CJ, Hartkamp MJ, Mali WP. Measuring blood flow by nontriggered 2D phase-contrast MR angiography. Magn Reson Imaging 1996;14:609-614.

12. Tarnawski M, Padayachee S, West DJ, et al. The measurement of time-averaged flow by magnetic resonance imaging using continuous acquisition in the carotid arteries and its comparison with Doppler ultrasound. Clin Phys Physiol Meas 1990;11:27-36.

13. Bateman GA. Vascular compliance in normal pressure hydrocephalus. Am J Neuroradiol 2000;21:1574-1585.
14. Bateman GA. Association between arterial inflow and venous outflow in idiopathic and secondary intracranial hypertension. J Clin Neurosci 2006;13:550-556.

15. Bateman GA. Vascular hydraulics associated with idiopathic and secondary intracranial hypertension. AJNR Am J Neuroradiol 2002;23:1180-1186.

16. Evans AJ, Iwai F, Grist TA, et al. Magnetic resonance imaging of blood flow with a phase subtraction technique: in vitro and in vivo validation. Invest Radiol 1993;28:109-115.

17. Laffon $\mathrm{E}$, Valli N, Latrabe V, et al. A validation of a flow quantification by MR phase mapping software. Eur J Radiol 1998;27: $166-172$.

18. Powell AJ, Maier SE, Chung T, et al. Phase-velocity cine magnetic resonance imaging measurement of pulsatile blood flow in children and young adults: in vitro and in vivo validation. Pediatr Cardiol 2000;21:104-110.

19. Kim J, Thacker NA, Bromiley NA, et al. Prediction of the Jugular Waveform using a model of CSF dynamics. Am J Neuroradiol 2007;28:983-989.

20. Felgenhauer K. Protein size and cerebrospinal fluid composition. Klin Wochenschr 1974;52:1158-1164.

21. Greitz D. Radiological assessment of hydrocephalus: new theories and implications for therapy. Neurosurg Rev 2004;27:145-165.

22. Dandy WE, Blackfan WD. Internal hydrocephalus: an experimental, clinical and pathological study. Am J Dis Child 1914;8:406481 .

23. Greitz D. On the active vascular absorption of plasma proteins from tissue: rethinking the role of the lymphatic system. Med Hypotheses 2002;59:696-702.

24. Vanneste JA. Diagnosis and management of normal-pressure hydrocephalus. J Neurol 2000;247:5-14.

25. Vanneste JA. Three decades of normal pressure hydrocephalus: are we wiser now? J Neurol Neurosurg Psychiatry 1994;57:10211025.

26. Vanneste J, Van Acker R. Normal pressure hydrocephalus: did publications alter management? J Neurol Neurosurg Psychiatry 1990;53:564-568.

27. Kellie G. An account of the appearances observed in the dissection of two of the three individuals presumed to have perished in the storm of the $3 \mathrm{rd}$, and whose bodies were discovered in the vicinity of Leith on the morning of the 4th November 1821, with some reflections on the pathology of the brain. Trans Medico-Chirurg Soc Edinb 1824;1:84-169.

28. Monro A. Observations on the structure and function of the nervous system. Edinburgh: Creech and Johnson, 1823.

29. Cushing H. The third circulation. In: Studies in intracranial physiology and surgery. London: Oxford University Press, London 1926:1-51.

30. Greitz D, Greitz T, Hindmarsh T. A new view on the CSF-circulation with the potential for pharmacological treatment of childhood hydrocephalus. Acta Paediatr 1997;86:125-132.

31. Stolz E, Kaps M, Kern A, et al. Transcranial color-coded duplex sonography of intracranial veins and sinuses in adults: reference data from 130 volunteers. Stroke 1999;30:1070-1075.

32. Greitz D, Hannerz J, Rahn T, et al. MR imaging of cerebrospinal fluid dynamics in health and disease: on the vascular pathogenesis of communicating hydrocephalus and benign intracranial hypertension. Acta Radiol 1994;35:204-211.

33. Greitz D, Hannerz J, Bellander J, et al. Restricted arterial expansion as a universal causative factor in communicating hydrocephalus. In: Proceedings of the XV Symposium Neuroradialogicum. Takahashi M, Korogi Y, Moseley I, editors. Heidelberg: Springer Verlag Berlin, 1995:14-18.

34. Greitz D, Greitz T. The pathogenesis and hemodynamics of hydrocephalus: a proposal for a new understanding. Int J Neuroradiol 1997;3:367-375.

35. Greitz D. The hydrodynamic hypothesis versus the bulk flow hypothesis. Neurosurg Rev 2004;27:299-300.

36. Greitz D. Cerebrospinal fluid circulation and associated intracranial dynamics: a radiologic investigation using MR imaging and radionuclide cisternography. Acta Radiol Suppl 1993;386:1-23.

37. Hakim S. Biomechanics of hydrocephalus. Acta Neurol Latinoam 1971;1(Suppl 1):169-194. 
38. Hakim S. Considerations on the physics of hydrocephalus and its treatment. Exp Eye Res 1977;25(Suppl):391-399.

39. Hakim S, Venegas JG, Burton JD. The physics of the cranial cavity, hydrocephalus and normal pressure hydrocephalus: mechanical interpretation and mathematical model. Surg Neurol 1976;5:187-210

40. Johnston I, Paterson A. Benign intracranial hypertension. I. Diagnosis and prognosis. Brain 1974;97:289-300.

41. Johnston I, Paterson A. Benign intracranial hypertension. II. CSF pressure and circulation. Brain 1974;97:301-312.

42. Biousse V, Ameri A, Bousser MG. Isolated intracranial hypertension as the only sign of cerebral venous thrombosis. Neurology 1999;53:1537-1542.

43. Gross CE, Tranmer BI, Adey G, et al. Increased cerebral blood flow in idiopathic pseudotumour cerebri. Neurol Res 1990;12: $226-230$

44. Foley J. Benign forms of intracranial hypertension; toxic and otitic hydrocephalus. Brain 1955;78:1-41.

45. Breteler MM, Van Swieten JC, Bots ML, et al. Cerebral white matter lesions, vascular risk factors, and cognitive function in a population-based study: the Rotterdam Study. Neurology 1994;44: $1246-1252$.

46. Lindgren A, Roijer A, Rudling O, et al. Cerebral lesions on magnetic resonance imaging, heart disease, and vascular risk factors in subjects without stroke: a population-based study. Stroke 1994;25: 929-934.

47. Klassen AC, Sung JH, Stadlan EM. Histological changes in cerebral arteries with increasing age. J Neuropathol Exp Neurol 1968; 27:607-623.

48. Furuta A, Ishii N, Nishihara Y, et al. Medullary arteries in aging and dementia. Stroke 1991;22:442-446.

49. Ravens JR. Vascular changes in the human senile brain. Adv Neurol 1978;20:487-501.

50. Hommel M, Gray F. Microvascular pathology. In: Caplan LR, editor. Brain ischaemia: basic concepts and clinical relevance. Vol. 1. New York: Springer-Verlag, 1995:215-223.

51. Poirier J, Derouesne C. Cerebral lacunae: a proposed new classification. Clin Neuropathol 1984;3:266.

52. Kapeller P, Barber R, Vermeulen RJ, et al. Visual rating of agerelated white matter changes on magnetic resonance imaging: scale comparison, interrater agreement, and correlations with quantitative measurements. Stroke 2003;34:441-445.

53. Fazekas F, Barkhof F, Wahlund LO, et al. CT and MRI rating of white matter lesions. Cerebrovasc Dis 2002;13(Suppl 2):31-36.

54. Patankar TF, Mitra D, Varma A, et al. Dilatation of the Virchow-
Robin space is a sensitive indicator of cerebral microvascular disease: study in elderly patients with dementia. Am J Neuroradiol 2005;26:1512-1520.

55. Naish JH, Baldwin RC, Patankar T, et al. Abnormalities of CSF flow patterns in the cerebral aqueduct in treatment-resistant latelife depression: a potential biomarker of microvascular angiopathy. Magn Reson Med 2006;56:509-516.

56. Kalaria RN. The role of cerebral ischemia in Alzheimer's disease. Neurobiol Aging 2000;21:321-330.

57. Kivipelto M, Helkala EL, Laakso MP, et al. Midlife vascular risk factors and Alzheimer's disease in later life: longitudinal, population based study. BMJ 2001;322:1447-1451.

58. Skoog I, Lernfelt B, Landahl S, et al. 15-year longitudinal study of blood pressure and dementia. Lancet 1996;347:1141-1145.

59. Hofman A, Ott A, Breteler M, et al. Atherosclerosis, apolipoprotein E, and prevalence of dementia and Alzheimer's disease in the Rotterdam Study. Lancet 1997;349:151-154.

60. Snowdon DA. Aging and Alzheimer's disease: lessons from the Nun Study. Gerontologist 1997;37:150-6.

61. Frolich L, Klinger T, Berger FM. Treatment with donepezil in Alzheimer patients with and without cerebrovascular disease. J Neurol Sci 2002;203-204:137-139.

62. De Leeuw FE, Barkhof F, Scheltens P. Alzheimer's disease-one clinical syndrome, two radiological expressions: a study on blood pressure. J Neurol Neurosurg Psychiatry 2004;75:1270-1274.

63. Simons M, Schwarzler F, Lutjohann D, et al. Treatment with simvastatin in normocholesterolemic patients with Alzheimer's disease: a 26-week randomized, placebo-controlled, double-blind trial. Ann Neurol 2002;52:346-350.

64. Grobe-Einsler R, Traber J. Clinical results with nimodipine in Alzheimer disease. Clin Neuropharmacol 1992;15(Suppl 1 Pt A): 416A-417A.

65. Lopez-Arrieta JM, Birks J. Nimodipine for primary degenerative, mixed and vascular dementia. Cochrane Database Syst Rev 2002; 3:CD000147.

66. Silverberg GD, Levinthal E, Sullivan EV, et al. Assessment of low-flow CSF drainage as a treatment for AD: results of a randomized pilot study. Neurology 2002;59:1139-1145.

67. Bateman GA, Levi CR, Schofield P, et al. Quantitative measurement of cerebral haemodynamics in early vascular dementia and Alzheimer's disease. J Clin Neurosci 2006;13:563-568.

68. Bateman GA. The role of altered impedance in the pathophysiology of normal pressure hydrocephalus, Alzheimer's disease and syringomyelia. Med Hypotheses 2004;63:980-985. 